This document is confidential and is proprietary to the American Chemical Society and its authors. Do not copy or disclose without written permission. If you have received this item in error, notify the sender and delete all copies.

\title{
Surfaces with dual functionality through specific co- immobilization of self-assembled polymeric nanostructures
}

\begin{tabular}{|r|l|}
\hline Journal: & Langmuir \\
\hline Manuscript ID & la-2018-02812t.R1 \\
\hline Manuscript Type: & Article \\
\hline Date Submitted by the & $21-$ Sep-2018 \\
\hline Complete List of Authors: & $\begin{array}{l}\text { Rigo, Serena; University of Basel, Department of Chemistry } \\
\text { Gunkel-Grabole, Gesine; University of Basel, Department of Chemistry } \\
\text { Meier, Wolfgang; University of Basel, Department of Chemistry } \\
\text { Palivan, Cornelia; University of Basel, Department of Chemistry }\end{array}$ \\
\hline
\end{tabular}




\title{
Surfaces with dual functionality through specific co- immobilization of self-assembled polymeric
}

\author{
nanostructures
}

\author{
Serena Rigo, Gesine Gunkel-Grabole, Wolfgang Meier, Cornelia G. Palivan* \\ Department of Chemistry, University of Basel, Mattenstrasse 24a, BPR 1096, CH-4002 Basel, \\ Switzerland
}




\begin{abstract}
Co-immobilization of functional, nano-sized assemblies broadens the possibility to engineer dually functionalized active surfaces with nanostructured texture. Surfaces decorated with different nano-assemblies, such as micelles, polymersomes or nanoparticles are in high demand for various applications ranging from catalysis, biosensing up to antimicrobial surfaces. Here, we present a combination of bio-orthogonal and catalyst free strain promoted Azide-Alkyne click (SPAAC) and the thiol-ene reactions to simultaneously co-immobilize various nano-assemblies; we selected polymersome - polymersome and polymersome - micelle assemblies. For the first time, the immobilization method using SPAAC reaction was studied in detail to attach soft, polymeric assemblies on a solid support. Together, the SPAAC, and thiol-ene reactions successfully co-immobilized two unique self-assembled structures on the surfaces. Additionally, polydimethylsiloxane (PDMS) based polymersomes were used as "ink" for direct immobilization from a PDMS based micro stamp onto a surface creating locally defined patterns. Combining immobilization reactions has the advantage to attach any kind of nano-assembly pairs, resulting in surfaces with "desired" interfacial properties. Different nano-assemblies which encapsulate multiple active compounds co-immobilized on a surface will pave the way for the development of multifunctional surfaces with controlled properties and efficiency.
\end{abstract}

Keywords: Co-immobilization, polymersome, micelle, bifunctionalization, Strain promoted Azide-Alkyne Click reaction, Thiol-ene reaction 


\section{INTRODUCTION}

Specialized surfaces implementing specific properties or functionalities on solid supports (e.g. hydrophilic, hydrophobic, reflective, non-adhesive, anti-fouling, drug releasing surfaces etc.) ${ }^{1}$ play an important role in everyday life: they support novel properties like non-sticking surfaces in pans, water repellent surfaces for car windows, ${ }^{2}$ or anti-fouling surfaces for medical applications. ${ }^{3,4}$ The orthogonal immobilization of active molecules ${ }^{5}$ is a largely proposed active strategy for medical uses such as inhibiting microbial growth (e.g. antimicrobial peptides ${ }^{6}$ or quaternary ammonium compounds ${ }^{7}$ ); however, unprotected compounds often suffer rapid degradation in complex biological environments, limiting their efficacy to short time periods. Polymeric nano-assemblies (e.g. polymersomes ${ }^{8}$, micelles, nanoparticles ${ }^{9}$ ) are ideally suited to protect such active agents by shielding them from harsh environments, or by controlling their release profile ${ }^{10}$ or location. ${ }^{11,12}$

Polymeric nano-assemblies have been designed with external functional groups ${ }^{13}$ to allow for their immobilization through both non-covalent and covalent interactions onto functionalized surfaces, that expose complementary groups often introduced through silane chemistry. ${ }^{14,} 15$ Some examples of non-covalent interactions include molecular recognition pairs such as biotinstreptavidin, ${ }^{12,} 16$ or electrostatic interactions between oppositely charged surfaces and particles. ${ }^{17,}{ }^{18}$ Covalent binding is generally more robust and can be implemented by various different reactions including: Schiff-base formation between aldehyde and amine ${ }^{19}, 20$ with possible further reductive amination, ${ }^{11,21}$ thiol-ene reactions between thiol and an alkene, ${ }^{14}$ or triazole formation through azide alkyne click chemistry. ${ }^{22,}{ }^{23}$ A particularly appealing approach for covalent immobilization of polymer nano-assemblies is the catalyst free SPAAC reaction, which relies on cyclooctynes with high ring strain, because it overcomes the major toxicity 
drawback of copper catalyzed click reactions allowing it to be used in vitro and in vivo. ${ }^{24,25} 26,27$ It has also been used previously to modify various nano-assemblies, such as liposomes ${ }^{28,}{ }^{29}$, nanoparticles ${ }^{30}$, quantum dots, ${ }^{31}$ or polymersomes ${ }^{32}$. Despite its advantages over other methods and high biocompatibility, to the best of our knowledge the SPAAC reaction has not been used to immobilize soft polymer assemblies, to improve the application of surfaces in medicine.

Soft lithography techniques offer an efficient approach to generate patterned functionalized surfaces. In particular, micro-contact printing $(\mu \mathrm{CP})$ using PDMS micro-stamps has served to produce various patterns of self-assembled monolayers, ${ }^{33-35}$ on which for instance liposomes were immobilized. $^{36,37}$ The SPAAC reaction has also been used to create surface patterns by $\mu \mathrm{CP}$ a cyclooctyne linker on an azide terminated monolayer. ${ }^{38}$ In this fashion, liposomes, ${ }^{39,40}$ or inorganic nanoparticles ${ }^{41-43}$ have also been used as "ink" on PDMS stamps for direct immobilization. However, $\mu \mathrm{CP}$ of polymeric, PDMS based nano-assemblies has yet to be investigated.

Here, we present a method to use dually functionalized surfaces to simultaneously immobilize two unique nano-assemblies, or to perform $\mu \mathrm{CP}$ to obtain controlled patterns on the surface. We selected two distinct methods for covalent co-immobilization of nano-assemblies: SPAAC and thiol-ene click reactions. ${ }^{14}$ Polymersomes and micelles, known to provide protection of encapsulated biologically relevant molecules, were decorated with specific molecular functionalities to allow surface immobilization. All nano-assemblies were formed by the selfassembly of tri block copolymers composed of poly(2-methyl-2-oxazoline) (PMOXA) and PDMS as the hydrophilic and hydrophobic domains respectively. ${ }^{44-46}$ For the first time, immobilization of polymeric nano-assemblies through the SPAAC reaction was implemented using polymersomes containing azide functional groups and the thiol-ene reaction using either 
polymersomes or micelles containing methacrylate functionalized polymers. ${ }^{14}$ The polymersomes were characterized by light scattering (LS), transmission electron microscopy (TEM), and fluorescence correlation spectroscopy (FCS) prior to their immobilization. The immobilization of azide containing polymersomes on dibenzocyclooctyne (DBCO) functionalized surfaces was studied in detail, while the immobilization via the thiol-ene reaction was performed in conditions previously established by our group. ${ }^{14}$ In order to generate patterns of nano-assemblies, the polymersomes were used as "ink" for $\mu \mathrm{CP}$ of the surface through covalent immobilization using a stamp made out of PDMS. To enlarge the range of surface morphologies and underline the applicability of the methods presented, polymersomes and micelles served for the generation of dually functionalized surfaces. Immobilization of these nano-assemblies on a solid support were visualized by the combination of confocal laser scanning microscopy (CLSM), where the nanostructures were tagged with fluorescent dyes, atomic force microscopy (AFM), and scanning electron microscopy (SEM).

Our approach broadens the possibility of simultaneously immobilizing two nanostructures loaded with active molecules to engineer active surfaces with dual functionality. This is particularly appealing because it allows for the attachment of any type, or combination of, nanoassemblies entrapping active molecules resulting in dually functionalized nanostructured interfaces with the environment.

\section{EXPERIMENTAL SECTION}

\section{Materials}

Methacrylate terminated $\mathrm{PMOXA}_{25}-b-\mathrm{PDMS}_{54}-b-\mathrm{PMOXA}_{25} \quad\left(\mathrm{M}_{\mathrm{n}}=8400 \mathrm{~g} \mathrm{~mol}^{-1}\right) \quad$ and $\mathrm{PMOXA}_{19}-b-\mathrm{PDMS}_{67}-b-\mathrm{PMOXA}_{19}\left(\mathrm{M}_{\mathrm{n}}=8000 \mathrm{~g} \mathrm{~mol}^{-1}, \mathrm{Ð}=1.3\right)$ were purchased from Polymer 
Source Inc.; bodipy 630/650, alexa fluor 488 azide, and Menzler glass cover slips $(22 \times 32 \mathrm{~mm})$, from Thermo Scientific Inc.; silica wafers $(\mathrm{P}<100>)$ from Si-Mat Germany; the microstamp from Research Micro Stamps; (3-aminoprpoyl)triethoxysilane (APTES) from Acros organics; ethyltrimethoxysilane from Alfa Aesar, Dicyclohexylcarbodiimide (DCC), and Ethyl acetate from Fluka Analytical; 1-Hydroxy-6-chloro-benzotriazole (6-Cl-HOBt) from Iris Biotech $\mathrm{GmbH}$; sodium chloride $(\mathrm{NaCl})$, and sodium phosphate dibasic $\left(\mathrm{Na}_{2} \mathrm{HPO}_{4}\right)$ from $\mathrm{Merck}$; potassium chloride $(\mathrm{KCl})$, and potassium di-hydrogen phosphate $\left(\mathrm{KH}_{2} \mathrm{PO}_{4}\right)$ from PanReac AppliChem; dichloromethane (DCM) from VWR chemical; DBCO-Cy5, DBCO-acid, trimethylamine, toluene, ethanol, (3-Mercaptopropyl)trimethoxysilane, sodium hydroxide $(\mathrm{NaOH})$, and (tris(2-carboxyethyl)phosphine) (TCEP) from Sigma Aldrich. All chemicals were used as received unless stated otherwise.

\section{Polymer synthesis and characterization}

The block copolymers (1) $\mathrm{PMOXA}_{6}-b-\mathrm{PDMS}_{43}-b-\mathrm{PMOXA}_{6}$ and (2) azide terminated $\mathrm{PMOXA}_{7}-b-\mathrm{PDMS}_{40}-b-\mathrm{PMOXA}_{7}$ were synthesized, characterized, and fractioned by co-solvent fractionation as reported in detail earlier by Lörcher et. al. ${ }^{45}$

\section{Nano-assembly formation}

Five different triblock copolymers composed of PMOXA and PDMS were used in this study:

1) $\mathrm{PMOXA}_{6}-b-\mathrm{PDMS}_{43}-b$-PMOXA 6 together with 2) azide terminated $\mathrm{PMOXA}_{7}-b-\mathrm{PDMS}_{40}-b$ $\left.\mathrm{PMOXA}_{7}, 3\right)$ methacrylate terminated $\mathrm{PMOXA}_{25}-b-\mathrm{PDMS}_{54}-b-\mathrm{PMOXA}_{25}$ and 4) $\mathrm{PMOXA}_{19}{ }^{-}$ $\mathrm{PDMS}_{67}-\mathrm{PMOXA}_{19}$ together with 5) sulforhodamine B (SRB) labeled PMOXA $18-b-\mathrm{PDMS}_{76^{-}} b-$ $\mathrm{PMOXA}_{18}$ 
Polymersomes containing $0,0.1,1.0$, and $10 \mathrm{~mol} \%$ of azide terminated polymers were formed by thin film rehydration. A total of $0.5 \mu \mathrm{mol}$ polymer were dissolved in $100 \mu \mathrm{EtOH}$ and dried into a film on a rotary evaporator. The polymer film was rehydrated with $1 \mathrm{~mL}$ PBS (137 mM $\mathrm{NaCl}, 2.7 \mathrm{mM} \mathrm{KCl}, 10 \mathrm{mM} \mathrm{Na}_{2} \mathrm{HPO}_{4}$, and $1.8 \mathrm{mM} \mathrm{KH}_{2} \mathrm{PO}_{4}, \mathrm{pH}$ 7.4) and stirred overnight. The turbid solution was extruded 13 times on a $1 \mathrm{ml}$ syringe extruder through a $200 \mathrm{~nm}$ membrane (Nuclepore Trac-Etch membrane, Whatman ${ }^{\circledR}$ ) and purified by size exclusion chromatography using a Sepharose 2B column (length: $25 \mathrm{~cm}$, diameter: $1 \mathrm{~cm}$ ) connected to ÄKTAprime, detecting the polymersome elution at $280 \mathrm{~nm}$ (Figure S1) and the final concentration was estimated using concentration loaded divided by the dilution factor during purification.

Polymersomes and micelles with methacrylate groups were formed by thin film rehydration in the dark as described previously. ${ }^{14} 1 \mu \mathrm{mol}$ polymer was dissolved in $2 \mathrm{~mL} \mathrm{DCM}\left(0.5 \mu \mathrm{mol} \mathrm{ml}^{-1}\right)$ together with a drop (ca. $5 \mu \mathrm{l}$ ) of a $0.9 \mathrm{mM}$ solution of SRB labeled polymer resulting in ca. $0.5 \mathrm{~mol} \%$ labelled polymer. The polymer solution was dried to a film prior to its rehydration by stirring at room temperature over night with $2 \mathrm{~mL}$ PBS (pH 7.4).

\section{Physical characterization of the nano-assemblies}

Dynamic Light Scattering (DLS) was performed on a Zetasizer nano ZSP (Malvern Instruments) at $25^{\circ} \mathrm{C}$. The scattering was recorded at a backscattering angle of $173^{\circ} .20 \mu 1$ of polymersome solution was diluted in $500 \mu \mathrm{PBS}$. Static light scattering (SLS) (LS instruments) was performed with a $30 \mathrm{~mW}$ HeNe laser at $23{ }^{\circ} \mathrm{C}$ and target scattering intensity of $40 \mathrm{kHz}$ using nanostructure solutions containing $1 \mathrm{~mol} \%$ azide terminated polymers (concentration estimated based on initial concentration during formulation of $0.5 \mu \mathrm{mol} \mathrm{ml}^{-1} \hat{=} 2.25 \mathrm{mg} \mathrm{ml}^{-1}$ divided by a dilution factor of 3.4 during purification) diluted to $0.3,0.2$, and $0.1 \mathrm{mg} \mathrm{ml}^{-1}$. Each concentration 
was measured at scattering angles between $40^{\circ}$ and $110^{\circ}$ with an angular step of $10^{\circ}$ for $20 \mathrm{~s}$ each. DLS measurements at $90^{\circ}$ for $60 \mathrm{~s}$ were also made on the LS instrument for the lowest concentration.

TEM images were recorded on a Phillips CM100 at an acceleration voltage of $80 \mathrm{kV} .5 \mu \mathrm{l}$ nanostructure solution, diluted in PBS (1:7), were deposited on a glow discharged formvar and carbon coated copper grid. After 1 min the samples were blotted off, washed twice with $5 \mu 1$ $\mathrm{H}_{2} \mathrm{O}$, and once with $5 \mu 12 \%$ uranyl acetate before being negatively stained with $5 \mu 12 \%$ uranyl acetate for $10 \mathrm{~s}$.

\section{FCS characterization of polymersomes with azide functionalities}

FCS experiments (Zeiss LSM880) were performed with fluorescent DBCO-Cy5 and a He-Ne laser $(\lambda=633 \mathrm{~nm})$ using a main beam splitter (MBS 488/561/633). The pinhole diameter was set to $90 \mu \mathrm{m}$ and the system was calibrated using PBS containing $100 \mathrm{nM}$ of DBCO-Cy5. Three independent polymersome batches were prepared for each mol ratio of azide terminated polymer and reacted with DBCO-Cy5 while shaking (100 rpm) overnight at $37^{\circ} \mathrm{C}$, and measured with a laser power of $1 \%$. The amount of DBCO-Cy5 added was equal to the concentration of azide terminated block copolymer. For samples without azide terminated polymers, the amount of DBCO-Cy5 added was the same as for the polymersome sample containing $1 \mathrm{~mol} \%$ azide terminated polymers. Each measurement represented an average of 30 recordings of $10 \mathrm{~s}$ duration. The obtained autocorrelation curves were fit by a 3-D diffusion model (Equation 1) either for a single-component, when analyzing the free dye, or for two-components when analyzing the polymersomes reacted with the dye where the parameters for one component were constrained according to the recorded values of the free dye. ${ }^{47}$ 


$$
\begin{gathered}
G(\tau)=1+\frac{1}{N} * G_{t} * G_{d} \\
G_{t}(\tau)=1+\frac{T_{t}}{1-T_{t}} * e^{-\frac{\tau}{\tau_{t}}} \\
G_{d}(\tau)=\sum_{i=1}^{n} \frac{\Phi_{i}}{\left(1+\left(\frac{\tau}{\tau_{d, i}}\right)^{\alpha_{i}}\right) *\left(1+\left(\frac{\tau}{\tau_{d, i}}\right)^{\alpha_{i}} * \frac{1}{S^{2}}\right)}
\end{gathered}
$$

Where $\mathrm{N}$ is the average number of particles in the confocal volume, $\mathrm{G}_{t}(\tau)$ describes the molecules in the triplet state, $T_{t}$ represents the triplet fraction, and $\tau_{t}$ represents the triplet relaxation time. The term $\mathrm{G}_{\mathrm{d}}(\tau)$ describes the diffusion of 1 component $(\mathrm{n}=1)$ or for two components $(n=2)$ in three dimensions. Finally, $\tau_{d}$ represents the diffusion time, $\Phi$ the fractional intensities, $\mathrm{S}$ the structural parameter, and $\alpha$ the anomaly parameter.

$\tau_{\mathrm{t}}$ was fixed to $3 \mu \mathrm{s}$ and $\mathrm{S}$ to $5 . \tau_{\mathrm{d}}$ of DBCO-Cy5 was determined applying the one component system model to the measurements recorded of the free dye solution and the obtained values were used as parameters for the free dye in further measurements. Then the average $\tau_{\mathrm{d}}$ of the polymersomes interacting with the dye was determined using the two component system where the first component $\tau_{\mathrm{d}}$ was fixed to the respective average of free DBCO-Cy5.

\section{DBCO functionalized surfaces}

The surfaces of silicon wafers or glass slides were activated in an oxygen plasma (Plasma Cleaner, Harrick Plasma) for $5 \mathrm{~min}$ before being immersed in toluene (anhydrous, $25 \mathrm{~mL}$ ) containing APTES $(4.3 \mu \mathrm{L}, 18 \mu \mathrm{mol})$, ethyltrimethoxysilane $(4.3 \mu \mathrm{L}, 27 \mu \mathrm{mol})$, and triethylamine $(42 \mu \mathrm{L}, 0.3 \mathrm{mmol}) .{ }^{14}$ The surfaces were shaken at $80 \mathrm{rpm}$ overnight at room temperature. Subsequently, the surfaces were rinsed with toluene followed by ethanol and dried 
under a gentle stream of compressed air. Afterwards, the surfaces were further functionalized with DBCO. Therefore, $2 \mu \mathrm{mol}$ of DBCO-acid, DCC, and 6-Cl-HOBt were separately dissolved in $1 \mathrm{~mL}$ ethyl acetate before being mixed together and stirred at $0{ }^{\circ} \mathrm{C}$ for $1 \mathrm{~h}$. The APTES functionalized surfaces were immersed in $20 \mathrm{~mL}$ ethyl acetate and the cooled reaction mixture was added. They were shaken horizontally at $80 \mathrm{rpm}$ overnight at room temperature, to allow the peptide bond formation between the amine on the surface and the acid on the DBCO linker. Subsequently, the surfaces were rinsed with ethyl acetate, water, and ethanol, and then dried under a gentle stream of compressed air.

\section{DBCO/Thiol bifunctionalized surfaces}

After plasma treatment the glasses were immersed in a $0.4 \%(\mathrm{w} / \mathrm{w})$ of silanes in toluene $(1: 1: 1$ weight \% APTES, (3-Mercaptopropyl)trimethoxysilane, and ethyltrimethoxysilane) to achieve a $33 \%$ relative density of each silane. Subsequently, the surfaces were washed and the amine groups of the APTES used to further functionalize the surface with an acid-DBCO linker via peptide bond formation as previously described, but with a reduced reaction time of 1.5 hours in order to minimize any potential side reactions.

\section{Characterization of DBCO functionalized surfaces}

DBCO functionalized surfaces were characterized and compared to non-functionalized and amino (APTES) functionalized surfaces. Static water contact angles (KSV, instruments, CAM100 Version 2.1.1) were measured on at least 3 independently prepared surfaces. Attenuated total reflection - Fourier transformed infrared (ATR-FTIR) spectroscopy (Bruker Alpha, platinum-ATR) measurements were performed on the surfaces recording 24 scans. 
DBCO functionalized surfaces were immersed in $3.5 \mathrm{~mL}$ Alexafluor488-azide (900 nM in PBS) to react overnight at $37^{\circ} \mathrm{C}, 20 \mathrm{rpm}$. To visualize the surfaces, they were washed with PBS and laser scanning microscopy (LSM) (Zeiss LSM510 META/ConfocoCor 2 FCS microscope) was performed using an Argon2 $(\lambda=488 \mathrm{~nm})$ laser and a water immersion objective (C-Apochromat 40x/1.2 W). The pinhole diameter was set to $70 \mu \mathrm{m}$ and a main beam splitter (HFT488), a secondary beam splitter (NFT490) and a band pass filter (BP 505-550) where used throughout all experiments. All images were recorded with a detector gain fixed to 679. The brightness was adjusted equally for all images using ImageJ.

\section{Immobilization of polymersomes on DBCO functionalized surfaces}

A $10 \mathrm{mM}$ bodipy aliquot in DMSO was diluted in water to obtain a bodipy stock solution $(25 \mu \mathrm{M})$ and was added to azide functionalized polymersomes in PBS (final concentration of bodipy $=50 \mathrm{nM}$ ). Bodipy interacts with the PDMS block of the membrane due to non-specific hydrophobic interactions allowing the visualization of the vesicles. $400 \mu 1$ of this polymersome solution were added onto DBCO functionalized surfaces with an area of approximately $1 \mathrm{~cm}^{2}$. The SPAAC immobilization reaction was allowed to proceed overnight by shaking horizontally at $80 \mathrm{rpm}$ at room temperature. The surface was washed three times by immersion in $2 \mathrm{~mL}$ fresh PBS while shaking horizontally at $80 \mathrm{rpm}$ for $5 \mathrm{~min}$ at room temperature.

\section{Immobilization of polymersomes on DBCO/thiol bifunctionalized surfaces}

To immobilize nano-assemblies on bifunctionalized surfaces, a bifunctional surface containing DBCO and thiol moieties as well as two different nanostructures were used. Bodipy $(50 \mathrm{nM})$ was utilized to visualize the azide exposing polymersomes through hydrophobic interactions while 
the methacrylate nanostructures were visualized by the addition of SRB labeled polymers during the self-assembly process.

A microstamp of parallel stripes $(5 \mu \mathrm{m}$ width, $5 \mu \mathrm{m}$ spacing, $10 \mu \mathrm{m}$ tall $)$ was inked with a polymersome solution (azide or methacrylate exposing polymersomes) by spreading the solution on the stamp with a Q-cotton tip. The stamp was brought in contact with the bi-functionalized $\mathrm{DBCO} /$ thiol surface for $10 \mathrm{~min}$ and weighted with a glass petri dish lid (ca. $18 \mathrm{~g}$ ). The stamp was removed and the surface was rinsed with PBS. The stamp was then washed by rinsing it with water, ethanol and sonication in ethanol for $10 \mathrm{~min}$ before reuse.

The SRB labelled methacrylate nanostructure (polymersome and micelle) solution obtained after self-assembly was diluted 1:1 with PBS. TCEP $\left(20 \mathrm{mg} \mathrm{mL}^{-1}\right)$, the catalyst for the thiol-ene reaction was added to the polymersome solution at least $2 \mathrm{~h}$ prior to adjusting the $\mathrm{pH}$ to 7.0 with $2 \mathrm{M} \mathrm{NaOH}$. Afterwards, the methacrylate nanostructure suspension was mixed 1:1 with bodipy containing azide exposing polymersomes and $400 \mu 1$ of the mixture were added on the bifunctionalized surfaces of approximately $1 \mathrm{~cm}^{2}$. The immobilization reactions were allowed to proceed overnight while horizontally shaking at $80 \mathrm{rpm}$ at room temperature. Afterwards the surfaces were removed from the reaction solution and washed by immersion in $2 \mathrm{~mL}$ fresh PBS, while shaking horizontally at $80 \mathrm{rpm}$ for $5 \mathrm{~min}$ at room temperature (repeated $3 \mathrm{x}$ ).

\section{Characterization of immobilized nano-assemblies}

AFM (JPK Nanowizard 3, Version 6.0.63) was used to characterize the surfaces of immobilized polymersomes containing varying ratios of azides. A Tap150 Al-G cantilever (Budget Sensors, resonance frequency $150 \mathrm{kHz}$, force constant $5 \mathrm{~N} \mathrm{~m}^{-1}$ ) was used for AC mode measurements in PBS buffer. Images were analyzed with the JPK data processing software 
(Version spm_6.0.63). Further, these surfaces were characterized by SEM (Hitach S-4800) with an acceleration voltage of $5000 \mathrm{~V}$ at a working distance of $3.4 \mathrm{~mm}$. The surfaces were previously sputtered with platinum.

CLSM (Zeiss LSM880) was performed to visualize immobilized nanostructures. After immobilization the bodipy containing azide-polymersomes and the SRB labelled methacrylate polymersomes and micelles were visualized by CLSM in airyscan mode. A He-Ne laser $(\lambda=633$ $\mathrm{nm})$ was used to visualize bodipy containing vesicles, and a DBSS 561-10 Laser $(\lambda=561 \mathrm{~nm})$ for SRB labelled nano-assemblies. The images were recorded with an oil immersion objective lens (Plan Apochromat 63x/1.4 oil DIC M27), a mean beam splitter (MBS 488/561/633) and an airyscan detector. The laser power was kept as low as possible $(0.2 \%$ for $\lambda=561 \mathrm{~nm}, 2-20 \%$ for $\lambda=633 \mathrm{~nm}$ ) to minimize bleaching, the gain was always below 800 , and the pinhole diameter around $110 \mathrm{~nm}$ to be slightly above the minimal pinhole diameter for airyscan, which corresponds to 2.5 airy units and 2.7 airy units for $\lambda=633 \mathrm{~nm}$ and $\lambda=561 \mathrm{~nm}$, respectively. The images were airyscan processed with the Zeiss software ZEN $2.3 \mathrm{SP} 1$ and the brightness was further adjusted with the ImageJ software.

\section{RESULTS AND DISCUSSION}

\section{Formation of amphiphilic copolymer nano-assemblies}

Nano-assemblies exposing either azide or methacrylate functional groups at the interface with the environment were created by self-assembly of amphiphilic copolymers via thin film rehydration method to enable attachment on bi-functionalized surfaces. Different concentrations of azide groups were exposed on their surface by using different ratios of the $\mathrm{PMOXA}_{6}-b$ $\mathrm{PDMS}_{43}-b-\mathrm{PMOXA}_{6}$ and azide-terminated $\mathrm{PMOXA}_{7}-b-\mathrm{PDMS}_{40}-b-\mathrm{PMOXA}_{7}$ copolymers $(0.0$, 
$0.1,1.0$, and $10 \mathrm{~mol} \%$ ). The size distribution of the resulting assemblies was limited by extrusion and size exclusion chromatography (Figure S1). In order to promote the formation of different fluorescently labeled methacrylate exposing nano-assemblies two different methacrylate terminated copolymers $\left(\mathrm{PMOXA}_{19}-b-\mathrm{PDMS}_{67}-b-\mathrm{PMOXA}_{19}\right.$ to form polymersomes, or $\mathrm{PMOXA}_{25}-b-\mathrm{PDMS}_{54}-b-\mathrm{PMOXA}_{25}$ to form micelles) ${ }^{14}$ were mixed with traces of SRB labeled $\mathrm{PMOXA}_{18}-b-\mathrm{PDMS}_{76}-b-\mathrm{PMOXA}_{18}$

The assemblies containing increasing percentages of azide terminated block-copolymer were characterized by a combination of LS (DLS and SLS) and TEM. The different ratios of azide containing polymers did not have an influence on the size as an overall average diameter of all azide containing assemblies determined by DLS using the number particle size distribution (PSD) was $140 \pm 15 \mathrm{~nm}$ with polydispersities (PDI) ranging from 0.03 to 0.23 (Figure 1). The nano-assembly containing $1 \mathrm{~mol} \%$ azide terminated block-copolymer was analyzed in more detail to study the architecture by calculating the shape factor. We obtained a hydrodynamic radius $\left(R_{h}\right)$ of $84 \pm 7 \mathrm{~nm}$ and a radius of gyration $\left(R_{g}\right)$ of $76 \pm 1 \mathrm{~nm}$ (Figure S2) resulting in a shape factor $\left(\rho=R_{g} / R_{h}\right)$ of 0.9 , which is associated with a hollow spherical architecture. ${ }^{48}$ In addition, TEM micrographs show hollow spherical structures for all assemblies with similar sizes as determined by LS experiments (Figure 1, Figure S3).

Methacrylate functionalized polymers and the resulting nano-assemblies were generated in similar conditions as reported previously ${ }^{14}$. Methacrylate terminated PMOXA $_{19}-b-$ PDMS $_{67}-b$ $\mathrm{PMOXA}_{19}$ copolymers generated polymersomes, slightly larger (diameter of $180 \pm 95 \mathrm{~nm}$ by number PSD) and more polydisperse (PDI 0.32 up to 0.51) than those with azide functionalizations (Figure S4). The higher PDI is in agreement with the respective TEM micrographs, where deflated structures with a broader size distribution were observed (Figure 
S5). Micelles formed by methacrylate terminated $\mathrm{PMOXA}_{25}-b-\mathrm{PDMS}_{54}-b-\mathrm{PMOXA}_{25}$ copolymers appeared as round structures in TEM micrographs (Figure S6) with a smaller diameter of $80 \pm 50 \mathrm{~nm}$ obtained by number PSD (Figure S7) (PDI up to 0.43). As methacrylate terminated nano-assemblies cannot be purified by SEC due to interactions with the column, they were used directly, which explains their higher PDI compared to that of the azide containing polymersomes.
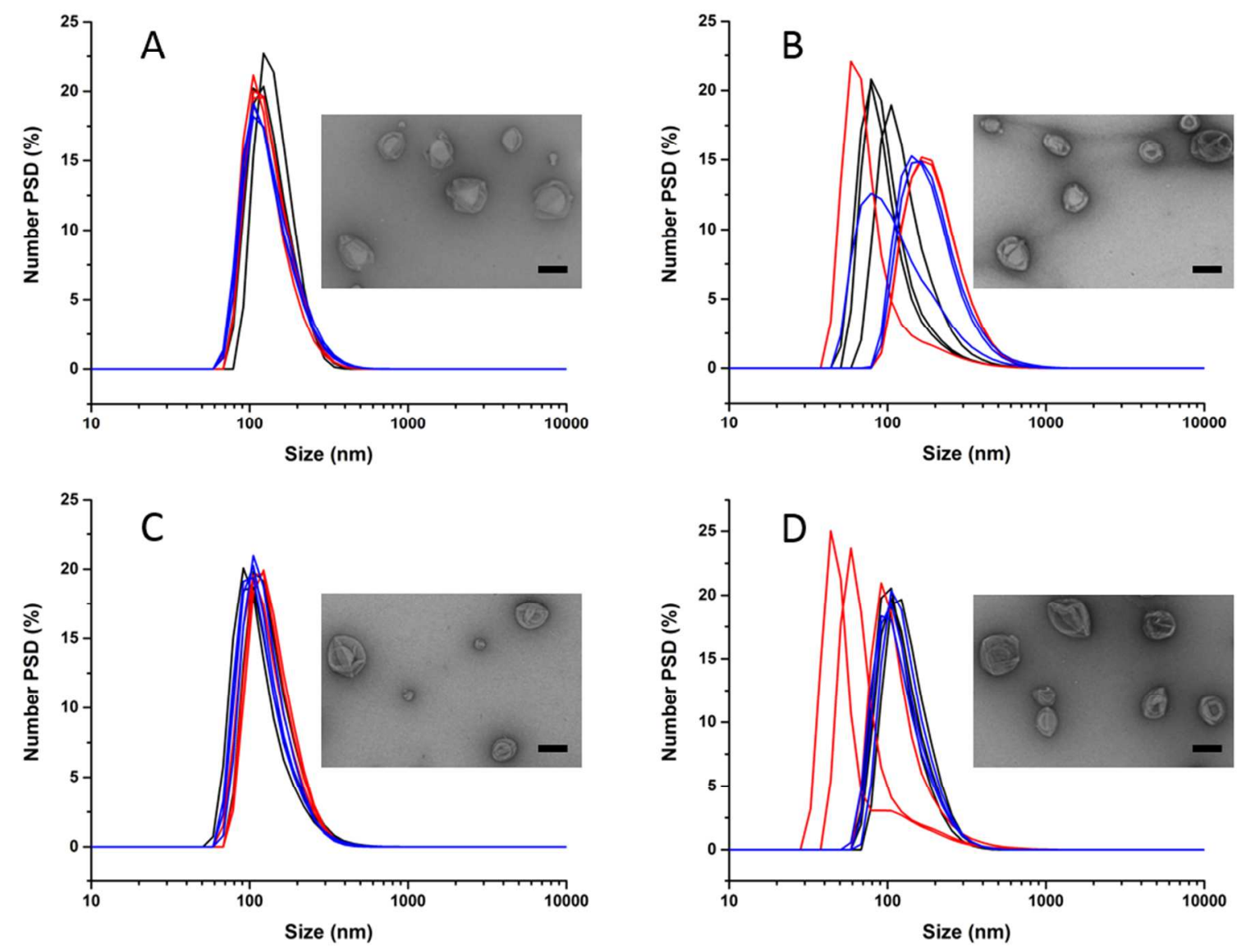

Figure 1. LS and TEM of azide-exposing nano-assemblies. DLS of polymersomes with different ratios of azide terminated polymers revealed similar diameters for the number average PSD: A) $0.0 \mathrm{~mol} \%, 139 \pm 4 \mathrm{~nm}$; B) $0.1 \mathrm{~mol} \%, 153 \pm 47 \mathrm{~nm}$; C) $1.0 \mathrm{~mol} \%, 132 \pm 7 \mathrm{~nm}$; D) $10 \mathrm{~mol} \%, 126 \pm 17 \mathrm{~nm}$. Three independent samples per polymersome ratio were measured three times each (black, red, and blue curves). Inserts are representative TEM images of the respective polymersomes, scale bars are $200 \mathrm{~nm}$. 
Accessibility of the azide moieties exposed on polymersomes was assessed by fluorescence correlation spectroscopy (FCS), which allows the study of the diffusion time of fluorescent molecules and their interactions with bigger assemblies such as nanoparticles, liposomes, or polymersomes. $9,47,49$ Fluorescently labeled DBCO (DBCO-Cy5) was reacted with azide functionalized polymersomes. The diffusion time $\left(\tau_{\mathrm{d}}\right)$, was determined by a two component fit where the first component's diffusion time was fixed to the one obtained for free DBCO and compared to free DBCO-Cy5 in solution determined by the one component fit (Table 1 and Figure 2). The increase of two orders of magnitude in $\tau_{\mathrm{d}}$ confirms that the DBCO-Cy5 molecule was associated with the azide-exposing polymersomes, which diffuse significantly slower through the confocal volume than the free fluorescent molecules. The small step in the autocorrelation curve (noted by a *) indicates the presence of a small fraction of polymersome clusters (Figure 2). Additionally, the $\mathrm{R}_{\mathrm{H}}$ calculated through the diffusion time is in accordance with the expected size of the polymersomes (Table 1). Polymersomes without azide groups were reacted with the same amount of DBCO-Cy5 as used for the polymersomes containing $1 \mathrm{~mol} \%$ azide terminated polymers served to establish the non-specific interaction of DBCO-Cy5 with non-functionalized polymersomes. (Figure 2). The fit of this autocorrelation curve resulted in a $73 \%$ population of free DBCO-Cy5 and a $27 \%$ fraction of slow diffusing particles, with $\tau_{\mathrm{d}} \approx 10^{\prime} 000 \mu \mathrm{s}$, which has been associated to a fraction of DBCO-Cy5 non-specifically interacting with unfunctionalized polymersomes, probably due to hydrophobic interactions with the polymeric membrane. The slow diffusion fractions of the fluorescent particles for azide functionalized polymersomes were in the majority (approaching $100 \%$ ), confirming an efficient bonding indicating that the azide groups were accessible on the polymersome surface (Table 1). 


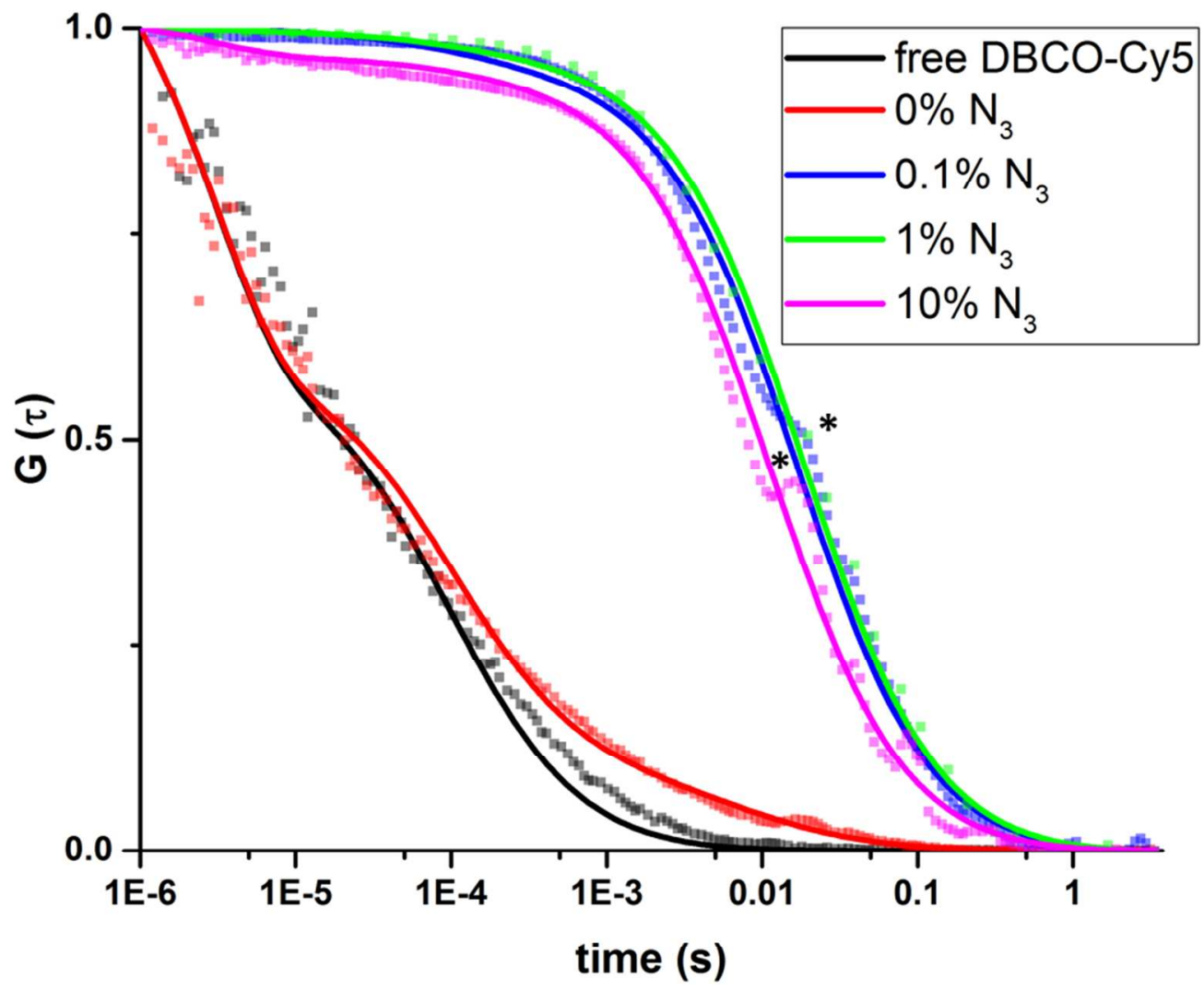

Figure 2. FCS of DBCO-Cy5 added to polymersomes containing different amounts of azide-terminated polymers. DBCO-Cy5 was added to the outside of the polymersomes and reacted overnight. The normalized autocorrelation curves for azide containing polymersomes showed a strong shift to higher diffusion times compared to azide free polymersomes (red line) or free DBCO-Cy5 (black line). The small step (*) in the autocorrelation curve is indicative for the presence of some clusters of polymersomes. 
Table 1. FCS parameters for each ratio of azide containing polymersomes.

\begin{tabular}{|c|c|c|c|c|c|}
\hline & \multirow{2}{*}{$\begin{array}{l}\text { Free } \\
\text { DBCO- } \\
\text { Cy5 }\end{array}$} & \multicolumn{4}{|c|}{$\begin{array}{l}\mathrm{PMOXA}_{6}-b-\mathrm{PDMS}_{43}-b-\mathrm{PMOXA}_{6} \text { polymersomes formed } \\
\text { with } \mathrm{x} \% \text { azide-terminated PMOXA } \mathrm{PM}_{7}-b-\mathrm{PDMS}_{40}-b \text {-PMOXA }\end{array}$} \\
\hline & & $0.0 \%$ & $0.1 \%$ & $1.0 \%$ & $10 \%$ \\
\hline diffusion time $[\mu \mathrm{s}]$ & $100 \pm 4$ & $9390 \pm 1200$ & $\begin{array}{ll}17630 & \pm \\
1050 & \end{array}$ & $21920 \pm 3830$ & $12580 \pm 3410$ \\
\hline $\begin{array}{l}\text { polymersome } \\
\text { fraction }[\%]\end{array}$ & $\mathrm{N} / \mathrm{A}$ & $27 \pm 9$ & $95 \pm 1$ & $95 \pm 3$ & $87 \pm 9$ \\
\hline $\mathrm{R}_{\mathrm{H}}[\mathrm{nm}]$ & $\mathrm{N} / \mathrm{A}$ & $54 \pm 7$ & $101 \pm 6$ & $126 \pm 22$ & $72 \pm 20$ \\
\hline
\end{tabular}

\section{Surface modification}

To immobilize azide containing polymersomes through the SPAAC reaction, surfaces were functionalized with DBCO in a two-step procedure (Figure 3A). First, surfaces were functionalized with primary amines using silane chemistry. A $40 \%$ relative density of amines on the surface was achieved by mixing the functional amine silane (APTES) and non-functional ethyl silane prior to the reaction with the surface. ${ }^{14}$ Secondly, DBCO functional groups were introduced via a peptide bond formation using a carboxy-functionalized DBCO. To further evaluate the functionalization of the surfaces the changes in surface hydrophobicity and ATRFTIR spectroscopy was studied. Amine modified surfaces possessed an increased hydrophobicity $\left(87 \pm 2^{\circ}\right)$ compared to non-functionalized surfaces $\left(67 \pm 1^{\circ}\right)$. Further functionalization with DBCO $\left(86 \pm 1^{\circ}\right)$ did not affect the hydrophobicity (Figure 3B). ATR-FTIR spectroscopy was performed after each functionalization step. A non-functionalized silicon wafer was used as background. DBCO functionalized surfaces show an inverted peak around $2200 \mathrm{~cm}^{-1}$ indicating the presence of the triple-bond ${ }^{50}$ compared with the non-functionalized or amine functionalized surface (Figure S8). Importantly, only surfaces functionalized with DBCO showed fluorescence 
after reaction with an azide functionalized fluorescent dye (Figure 3C-E and Figure S9). Therefore, the surfaces were successfully functionalized with DBCO, rendering them reactive towards azide containing molecules.
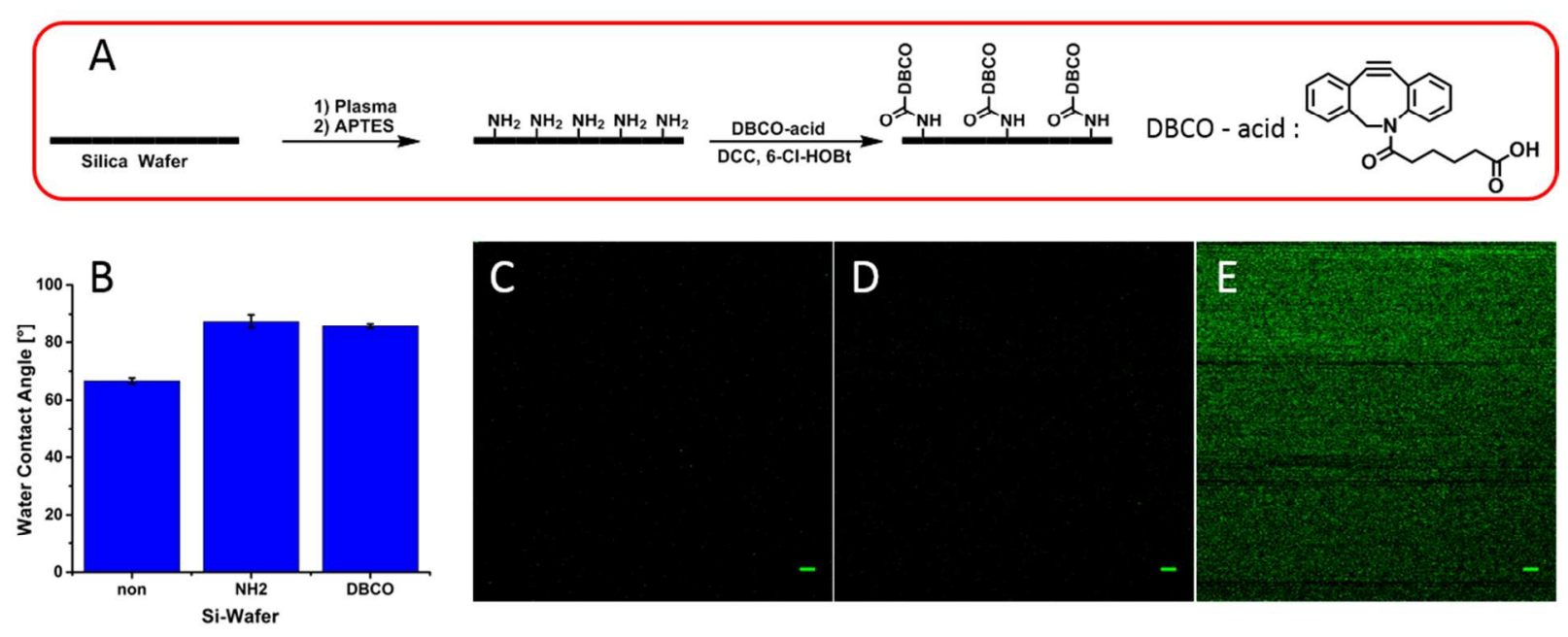

Figure 3. A) Schematic illustration of the two-step surface functionalization. B) Changes on static water contact angles of Si-Wafers during the different functionalization steps $(n=3)$. C-E) CLSM micrographs after reacting the surface during the different functionalization steps with Alexafluor488-azide: C) non-functionalized surface, $\mathrm{D}_{\text {) }} \mathrm{NH}_{2}$ functionalized surface, E) DBCO functionalized surface. Micrographs were recorded with the same settings and processed equally. Scale bars: $10 \mu \mathrm{m}$.

\section{Immobilization of azide-functionalized polymersomes on surfaces}

The SPAAC reaction was conducted to immobilize the azide exposing polymersomes onto the DBCO functionalized surface. After polymersome immobilization, the obtained surfaces were characterized by a combination of CLSM, SEM, and AFM. A hydrophobic fluorescent dye (Bodipy630/650) was adsorbed nonspecifically to the polymersomes to allow their detection by CLSM. Numerous round fluorescent objects were observed in the expected size range indicating successful polymersome immobilization on solid support by SPAAC. Furthermore, as already indicated by FCS, a few clusters of polymersomes were observed on the surfaces with an increased ratio of azide-terminated polymers. (Figure 4B and C). The images obtained by SEM 
(Figure S10A-C), were in agreement with the CLSM micrographs, and provided further evidence of the immobilization of azide containing polymersomes on a solid support. In addition, surfaces reacted with azide containing polymersomes showed round structures on the surface of $70-120 \mathrm{~nm}$ in height when observed by AFM while immersed in PBS buffer (Figure 4A-C). A significant $(p<0.05)$ difference in immobilization density between the azide-free $(0$ mol\%) polymersome and the azide-exposing polymersomes $(0.1,1.0$, or $10 \mathrm{~mol} \%)$ was detected; however, no difference between the polymersomes containing $0.1,1.0$, or $10 \mathrm{~mol} \%$ azide terminated polymers was observed (Table S1). Reacting azide-free polymersomes and DBCO functionalized surfaces revealed very few fluorescent dots by CLSM (Figure 4D), few immobilized vesicles by SEM (Figure S10D) and no structures of the expected heights by AFM (Figure 4D). Together, this data strongly suggests that the immobilization was due to the azide functional groups on the polymersome exterior.

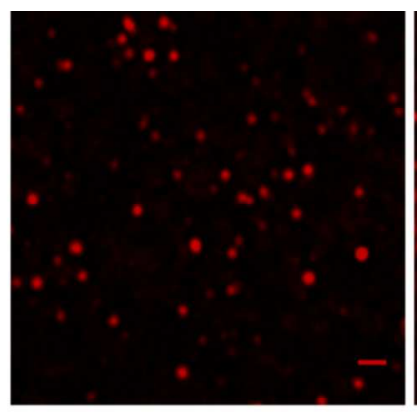

A

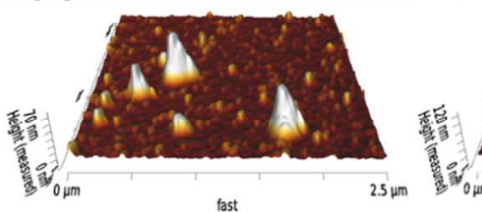

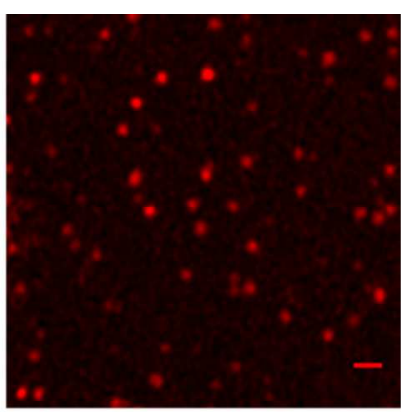

B

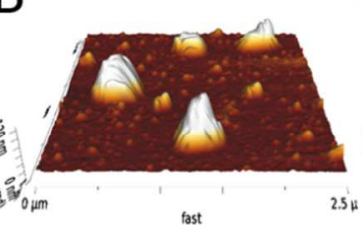

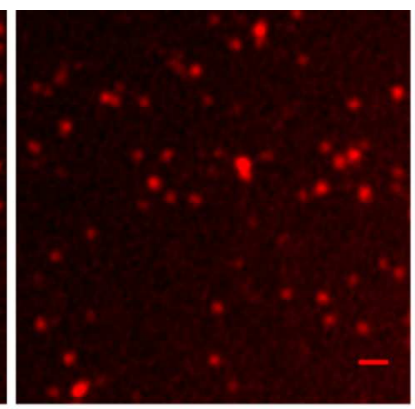

C
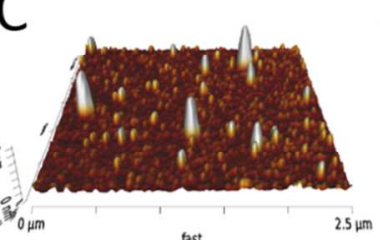

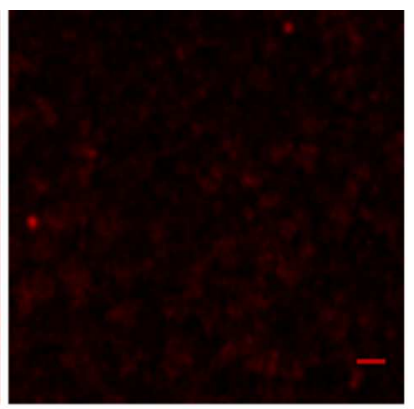

D

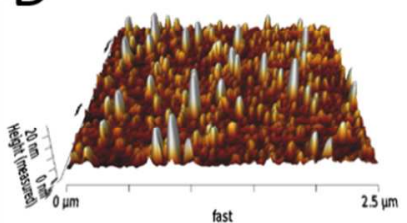

Figure 4. CLSM and AFM of immobilized polymersomes with different ratios of azide terminated polymers A) $0.1 \mathrm{~mol} \% \mathrm{~B}) 1.0 \mathrm{~mol} \% \mathrm{C}$ ) $10 \mathrm{~mol} \% \mathrm{D}) 0 \mathrm{~mol} \%$ on DBCO functionalized surfaces. Scale bars: $1 \mu \mathrm{m}$ for CLSM images. AFM scan size was $2.5 \mu \mathrm{m} \times 2.5 \mu \mathrm{m}$ each and the height scale bars are 70 (A), 120 (B), 70 (C) and $20 \mathrm{~nm}$ (D), respectively. 


\section{$\mu C P$ of polymersomes to obtain patterned surfaces}

It is attractive to create patterns of nano-assemblies for dually functionalized surfaces. Hence, $\mu \mathrm{CP}$ was used to immobilize self-assembled nanostructures. Polymersomes exposing azide groups and containing bodipy entrapped in their membrane were used as ink on a PDMS microstamp, transferred to the bifunctionalized surface, and visualized by CLSM. A clear striped pattern alternating between fluorescence and background every $5 \mu \mathrm{m}$ was observed (Figure 5A), indicating successful surface patterning. Further, the distinct fluorescent spots with clear borders observed in the patterned lines suggest that the main population of polymersomes and the minor population of polymersome clusters remained intact throughout the printing process and were transferred from the stamp to the surface. Similarly, polymersomes exposing methacrylate groups and labeled with SRB were successfully printed on the surface (Figure 5B). While the background-associated regions are clearly separated from the fluorescent stripes, there are few polymersomes present as well, probably due to non-specific attachment. The results further underline that PDMS-based nanostructures can be printed from a PDMS based stamp onto a functionalized surface. 

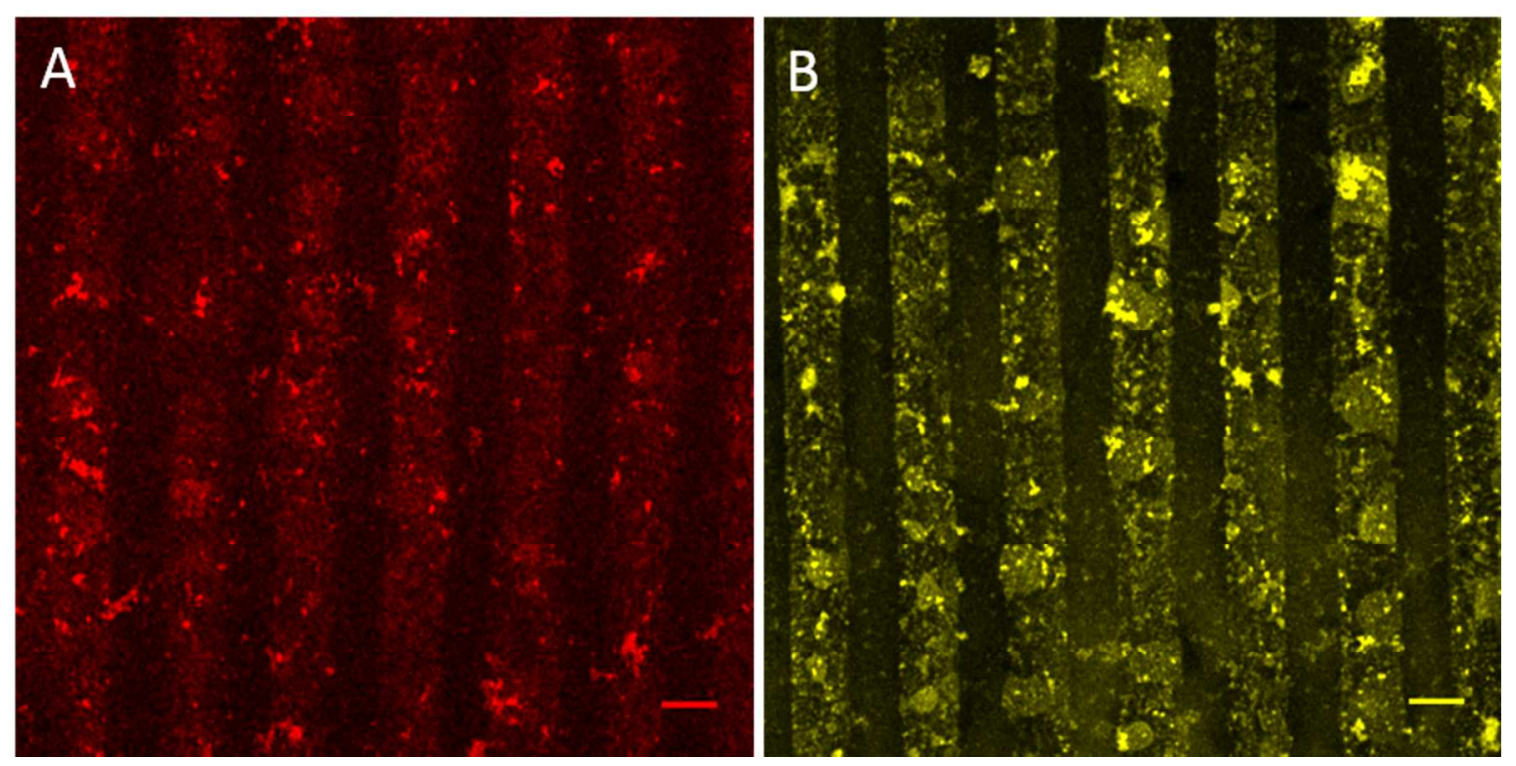

Figure 5. Polymersomes are immobilized within a pattern by $\mu \mathrm{CP}$ and observed with CLSM: A) Azidefunctionalized polymersomes stained with fluoresencent dye (Bodipy) and B) SRB labeled methacrylate functionalized polymersomes. Scale bars: $5 \mu \mathrm{m}$.

\section{Co-immobilization of two different nano-assemblies}

In order to obtain multifunctional surfaces, nanostructures with different functional groups were co-immobilize on the same solid support. Surfaces were functionalized with both, thiols to perform the thiol-ene reaction with methacrylate nanostructures ${ }^{14}$, and DBCO to perform the SPAAC reaction with azide containing polymersomes. Plasma activated silica wafers were reacted simultaneously to thiol and amine containing silanes prior to the condensation reaction between the amine and the DBCO-acid. In order to avoid any unwanted side reactions between thiols and alkynes, the condensation reaction time was reduced to 1.5 hours. The immobilization of the azide containing polymersomes was visualized through bodipy (red) interacting with the membrane while the immobilization of the methacrylate nanostructures was visualized by SRB (yellow). In addition, these fluorescent dyes served as models for biologically active low molecular weight molecules that can be integrated into the nano-assemblies. 
A mixture of the two differently functionalized polymersomes was added on the bifunctionalized surface. CLSM micrographs showed successful co-immobilization of the polymersomes due to the presence of both fluorescent signals associated to SRB and bodipy. (Figure 6A and S11A and B). The integrity of immobilized polymersomes was studied by ethanol treatment which would destroy the nano-assemblies and free the dye. Single polymersomes and few polymersome clusters of intact samples show a clear boarder. After ethanol addition the clear boarders disappeared and large agglomerations of material and fluorescence appeared. This comparison indicates that the nano-assemblies preserved their integrity upon immobilization (Figure S12). Therefore, the immobilization procedure does not affect the integrity of the polymersomes, which represent a key factor for further development of such bi-functionalized surfaces.

To go one step further in obtaining nanostructured, bi-functional surfaces we simultaneously immobilized two different assemblies: azide-exposing polymersomes (containing bodipy for visualization) and methacrylate-exposing micelles labelled with SRB. Azide exposing polymersomes were mixed equally with micelles exposing methacrylate groups, added to the bifunctionalized surface and reacted overnight via the SPAAC and the thiol-ene immobilization reaction. The immobilized structures were observed by CLSM as round fluorescent dots (Figure 6B and S11C and D). Furthermore, bodipy stained, azide exposing polymersomes were microcontact printed onto a bifunctionalized surface followed by a second immobilization of SRB-labelled methacrylate exposing micelles with the stamp rotated $90^{\circ}$ to create a checkerboard-pattern (Figure 6C). After patterning single nano-assemblies, it was shown that different nano-assemblies could be not only randomly co-immobilized on the same surface, but also in a distinct pattern. 

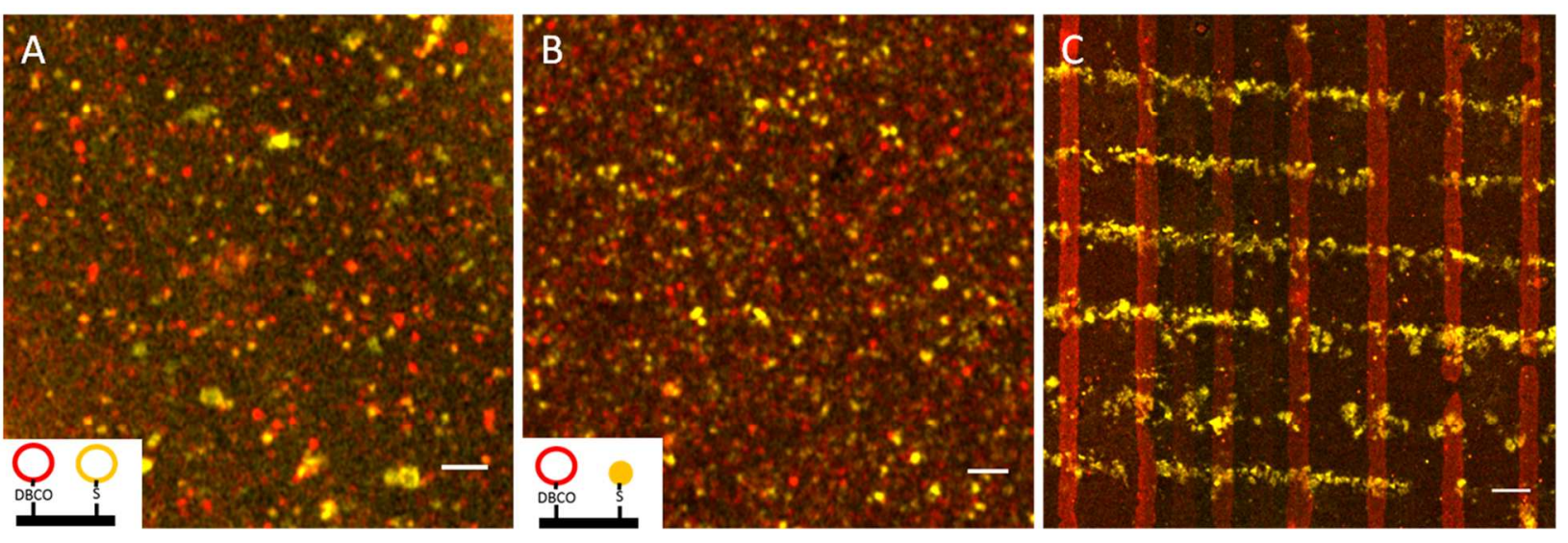

Figure 6. CLSM micrographs of azide functionalized polymersomes containing Bodipy co-immobilized with SRB labeled methacrylate functionalized nanostructures on DBCO and thiol bifunctionalized surfaces. A) Coimmobilization of azide-exposing polymersomes and methacrylate exposing polymersomes, B) Azide-exposing polymersomes and methacrylate-exposing micelles and C) Checkerboard pattern obtained by first printing azide exposing polymerosomes and then methacrylate exposing micelles with the stamp rotated by $90^{\circ}$. Scale bars: A and B) $2 \mu \mathrm{m} \mathrm{C)} 5 \mu \mathrm{m} . *$ hollow structure in the inset represents polymersomes and filled structures micelles.

\section{CONCLUSION}

Surfaces with nanostructured architecture and bi-functionality were successfully generated by the co-immobilization of azide containing polymersomes together with methacrylate exposing polymersomes or micelles on a solid support. To support simultaneous immobilization of the nano-assemblies, surfaces were bi-functionalized by using SPAAC and thiol-ene reactions, each of them serving to specifically bind nano-assemblies exposing appropriate functional groups (azide and methacrylate, respectively). The SPAAC reaction was analyzed in detail as it was used for the first time to immobilize polymeric nanostructures, while the thiol-ene immobilization reaction has been used in conditions already reported by our group. A comparison between polymersomes containing different ratios of azide terminated polymers $(0.1-10 \mathrm{~mol} \%)$ revealed that the ratio does not influence the polymersome immobilization, suggesting that only a few attachment points are required for their successful immobilization via 
the SPAAC reaction. Additionally, $\mu \mathrm{CP}$ was applied to immobilize polymersomes in a spatially defined pattern onto surfaces.

Patterned co-immobilization of different functionalized nano-assemblies to engineer multifunctional surfaces represents a general approach to induce a nanostructured architecture on a solid support and a dual functionality through the active compounds that can be entrapped inside these nano-assemblies. Permeability properties of the polymeric membranes of such nanoassemblies are maintained upon immobilization. ${ }^{11,12,21,51}$ Indeed, different cargos (as shown here by using dyes as models for biologically active low weight molecules) can be protected either in micelles or polymersomes. Such nanostructured surfaces with dual functionality are expected to improve the surface efficacy in applications, such as antimicrobial surfaces, or biosensing. 


\section{ASSOCIATED CONTENT}

\section{Supporting Information}

The following files are available free of charge.

Size exclusion chromatogram of azide containing polymersomes, additional TEM images, a Zimm plot, DLS and TEM of methacrylate containing nanostructures, IR spectrum of functionalized surfaces, SEM of immobilized vesicles and additional CLSM images of coimmobilized nanostructures on surfaces.

\section{AUTHOR INFORMATION}

\section{Corresponding Author}

* E-Mail: cornelia.palivan@unibas.ch

\section{ACKNOWLEDGMENT}

The authors would like to acknowledge the generous financial support from the SNSF, NCCRMSE and University of Basel. G. G.-G. gratefully acknowledges a postdoctoral research fellowship of the German Academic Exchange Service (DAAD). S.R. thanks Dr. Samuel Lörcher for the polymer synthesis and Susanne Erpel for help in SEM. The authors acknowledge Dr. Jason Duskey for editing the manuscript. 


\section{REFERENCES}

1. Zhao, N.; Wang, Z.; Cai, C.; Shen, H.; Liang, F. Y.; Wang, D.; Wang, C. Y.; Zhu, T.; Guo, J.; Wang, Y. X.; Liu, X. F.; Duan, C. T.; Wang, H.; Mao, Y. Z.; Jia, X.; Dong, H. X.; Zhang, X. L.; Xu, J., Bioinspired Materials: from Low to High Dimensional Structure. Adv Mater 2014, 26 (41), 6994-7017.

2. Han, Z. W.; Mu, Z. Z.; Yin, W.; Li, W.; Niu, S. C.; Zhang, J. Q.; Ren, L. Q., Biomimetic multifunctional surfaces inspired from animals. Adv Colloid Interfac 2016, 234, 27-50.

3. Glinel, K.; Thebault, P.; Humblot, V.; Pradier, C. M.; Jouenne, T., Antibacterial surfaces developed from bio-inspired approaches. Acta Biomater 2012, 8 (5), 1670-1684.

4. Rigo, S.; Cai, C.; Gunkel-Grabole, G.; Maurizi, L.; Zhang, X.; Xu, J.; Palivan, C. G., Nanoscience-Based Strategies to Engineer Antimicrobial Surfaces. Advanced Science 2018, 1700892.

5. Uth, C.; Zielonka, S.; Horner, S.; Rasche, N.; Plog, A.; Orelma, H.; Avrutina, O.; Zhang, K.; Kolmar, H., A Chemoenzymatic Approach to Protein Immobilization onto Crystalline Cellulose Nanoscaffolds. Angew Chem Int Edit 2014, 53 (46), 12618-12623.

6. Cao, P.; Yuan, C. Q.; Xiao, J. F.; He, X. Y.; Bai, X. Q., A biofilm resistance surface yielded by grafting of antimicrobial peptides on stainless steel surface. Surf Interface Anal 2018, $50(4), 516-521$.

7. Pant, J.; Gao, J.; Goudie, M. J.; Hopkins, S. P.; Locklin, J.; Handa, H., A multi-defense strategy: Enhancing bactericidal activity of a medical grade polymer with a nitric oxide donor and surface-immobilized quaternary ammonium compound. Acta Biomater 2017, 58, 421-431.

8. $\quad$ Discher, D. E.; Ahmed, F., Polymersomes. Annu Rev Biomed Eng 2006, 8, 323-341.

9. Najer, A.; Wu, D. L.; Nussbaumer, M. G.; Schwertz, G.; Schwab, A.; Witschel, M. C.; Schafer, A.; Diederich, F.; Rottmann, M.; Palivan, C. G.; Beck, H. P.; Meier, W., An amphiphilic graft copolymer-based nanoparticle platform for reduction-responsive anticancer and antimalarial drug delivery. Nanoscale 2016, 8 (31), 14858-14869.

10. Xiong, M. H.; Bao, Y.; Yang, X. Z.; Zhu, Y. H.; Wang, J., Delivery of antibiotics with polymeric particles. Adv Drug Deliver Rev 2014, 78, 63-76.

11. Langowska, K.; Kowal, J.; Palivan, C. G.; Meier, W., A general strategy for creating selfdefending surfaces for controlled drug production for long periods of time. J Mater Chem B 2014, 2 (29), 4684-4693.

12. Grzelakowski, M.; Onaca, O.; Rigler, P.; Kumar, M.; Meier, W., Immobilized ProteinPolymer Nanoreactors. Small 2009, 5 (22), 2545-2548.

13. Egli, S.; Schlaad, H.; Bruns, N.; Meier, W., Functionalization of Block Copolymer Vesicle Surfaces. Polymers-Basel 2011, 3 (1), 252-280.

14. Gunkel-Grabole, G.; Palivan, C.; Meier, W., Nanostructured Surfaces through Immobilization of Self-Assembled Polymer Architectures Using Thiol-Ene Chemistry. Macromol Mater Eng 2017, 302 (4).

15. Vutti, S.; Buch-Manson, N.; Schoffelen, S.; Bovet, N.; Martinez, K. L.; Meldal, M., Covalent and Stable CuAAC Modification of Silicon Surfaces for Control of Cell Adhesion. Chembiochem 2015, 16 (5), 782-791.

16. De Vries, W. C.; Tesch, M.; Studer, A.; Ravoo, B. J., Molecular Recognition and Immobilization of Ligand-Conjugated Redox-Responsive Polymer Nanocontainers. Acs Appl Mater Inter 2017, 9 (48), 41760-41766. 
17. Li, F.; Ketelaar, T.; Stuart, M. A. C.; Sudholter, E. J. R.; Leermakers, F. A. M.; Marcelis, A. T. M., Gentle immobilization of nonionic polymersomes on solid substrates. Langmuir 2008, 24 (1), 76-82.

18. Chen, Q.; de Groot, G. W.; Schonherr, H.; Vancso, G. J., Patterns of surface immobilized block copolymer vesicle nanoreactors. Eur Polym J 2011, 47 (2), 130-138.

19. Choi, J. M.; Yoon, B.; Choi, K.; Seol, M. L.; Kim, J. M.; Choi, Y. K., Micropatterning Polydiacetylene Supramolecular Vesicles on Glass Substrates using a Pre-Patterned Hydrophobic Thin Film. Macromol Chem Phys 2012, 213 (6), 610-616.

20. Domes, S.; Filiz, V.; Nitsche, J.; Fromsdorf, A.; Forster, S., Covalent Attachment of Polymersomes to Surfaces. Langmuir 2010, 26 (10), 6927-6931.

21. Zhang, X. Y.; Lomora, M.; Einfalt, T.; Meier, W.; Klein, N.; Schneider, D.; Palivan, C. G., Active surfaces engineered by immobilizing protein-polymer nanoreactors for selectively detecting sugar alcohols. Biomaterials 2016, 89, 79-88.

22. Rein, C.; Nissen, S.; Grzelakowski, M.; Meldal, M., Click-Chemistry of Polymersomes on Nanoporous Polymeric Surfaces. J Polym Sci Pol Chem 2016, 54 (13), 2032-2039.

23. Singh, M. S.; Chowdhury, S.; Koley, S., Advances of azide-alkyne cycloaddition-click chemistry over the recent decade. Tetrahedron 2016, 72 (35), 5257-5283.

24. Agard, N. J.; Prescher, J. A.; Bertozzi, C. R., A strain-promoted [3+2] azide-alkyne cycloaddition for covalent modification of biomolecules in living systems (vol 126, pg 15046, 2004). J Am Chem Soc 2005, 127 (31), 11196-11196.

25. Lutz, J. F., Copper-free azide-alkyne cycloadditions: New insights and perspectives. Angew Chem Int Edit 2008, 47 (12), 2182-2184.

26. Jewett, J. C.; Bertozzi, C. R., Cu-free click cycloaddition reactions in chemical biology. Chemical Society Reviews 2010, 39 (4), 1272-1279.

27. Chang, P. V.; Prescher, J. A.; Sletten, E. M.; Baskin, J. M.; Miller, I. A.; Agard, N. J.; Lo, A.; Bertozzi, C. R., Copper-free click chemistry in living animals. P Natl Acad Sci USA 2010, 107 (5), 1821-1826.

28. Bak, M.; Jolck, R. I.; Eliasen, R.; Andresen, T. L., Affinity Induced Surface Functionalization of Liposomes Using Cu-Free Click Chemistry. Bioconjugate Chem 2016, 27 (7), 1673-1680.

29. Blenke, E. O.; Klaasse, G.; Merten, H.; Pluckthun, A.; Mastrobattista, E., Liposome functionalization with copper-free "click chemistry". J Control Release 2015, 202, 14-20.

30. Wang, C. F.; Sarparanta, M. P.; Makila, E. M.; Hyvonen, M. L. K.; Laakkonen, P. M.; Salonen, J. J.; Hirvonen, J. T.; Airaksinen, A. J.; Santos, H. A., Multifunctional porous silicon nanoparticles for cancer theranostics. Biomaterials 2015, 48, 108-118.

31. Kotagiri, N.; Li, Z. Y.; Xu, X. X.; Mondal, S.; Nehorai, A.; Achilefu, S., Antibody Quantum Dot Conjugates Developed via Copper-Free Click Chemistry for Rapid Analysis of Biological Samples Using a Microfluidic Microsphere Array System. Bioconjugate Chem 2014, 25 (7), 1272-1281.

32. Gobbo, P.; Mossman, Z.; Nazemi, A.; Niaux, A.; Biesinger, M. C.; Gillies, E. R.; Workentin, M. S., Versatile strained alkyne modified water-soluble AuNPs for interfacial strain promoted azide-alkyne cycloaddition (I-SPAAC). J Mater Chem B 2014, 2 (13), 1764-1769.

33. Hammond, P. T.; Whitesides, G. M., Formation of Polymer Microstructures by Selective Deposition of Polyion Multilayers Using Patterned Self-Assembled Monolayers as a Template. Macromolecules 1995, 28 (22), 7569-7571. 
34. Cau, J. C.; Cerf, A.; Thibault, C.; Genevieve, M.; Severac, C.; Peyrade, J. P.; Vieu, C., Chemical engineering of silicon oxide surfaces using micro-contact printing for localizing adsorption events of nanoparticles, dendrimers and bacteria. Microelectron Eng 2008, 85 (5-6), $1143-1146$.

35. Qin, D.; Xia, Y. N.; Whitesides, G. M., Soft lithography for micro- and nanoscale patterning. Nat Protoc 2010, 5 (3), 491-502.

36. Shim, H. Y.; Lee, S. H.; Ahn, D. J.; Ahn, K. D.; Kim, J. M., Micropatterning of diacetylenic liposomes on glass surfaces. Mat Sci Eng C-Bio S 2004, 24 (1-2), 157-161.

37. Voskuhl, J.; Wendeln, C.; Versluis, F.; Fritz, E. C.; Roling, O.; Zope, H.; Schulz, C.; Rinnen, S.; Arlinghaus, H. F.; Ravoo, B. J.; Kros, A., Immobilization of Liposomes and Vesicles on Patterned Surfaces by a Peptide Coiled-Coil Binding Motif. Angew Chem Int Edit 2012, 51 (50), 12616-12620.

38. Wendeln, C.; Singh, I.; Rinnen, S.; Schulz, C.; Arlinghaus, H. F.; Burley, G. A.; Ravoo, B. J., Orthogonal, metal-free surface modification by strain-promoted azide-alkyne and nitrile oxide-alkene/alkyne cycloadditions. Chem Sci 2012, 3 (8), 2479-2484.

39. Mahajan, N.; Lu, R. B.; Wu, S. T.; Fang, J. Y., Patterning polymerized lipid vesicles with soft lithography. Langmuir 2005, 21 (7), 3132-3135.

40. Yu, S.; Kim, J. M.; Ahn, H., Micro-Contact Printing of Polydiacetylene Liposomes Using Hydrophilic Stamps. J Nanosci Nanotechno 2011, 11 (7), 6034-6038.

41. Santhanam, V.; Andres, R. P., Microcontact printing of uniform nanoparticle arrays. Nano Lett 2004, 4 (1), 41-44.

42. Platnich, C. M.; Banerjee, A.; Kollath, V. O.; Karan, K.; Trudel, S., Thiol-ene click microcontact printing of gold nanoparticles onto silicon surfaces. Can J Chem 2015, 96 (2), 190195.

43. Xin, Z. Q.; Liu, Y.; Li, X.; Liu, S. L.; Fang, Y.; Deng, Y. Q.; Bao, C.; Li, L. H., Conductive grid patterns prepared by microcontact printing silver nanoparticles ink. Mater Res Express 2017, 4 (1).

44. Nardin, C.; Hirt, T.; Leukel, J.; Meier, W., Polymerized ABA triblock copolymer vesicles. Langmuir 2000, 16 (3), 1035-1041.

45. Lorcher, S.; Meier, W., Cosolvent fractionation of PMOXA-b-PDMS-b-PMOXA: Bulk separation of triblocks from multiblocks. Eur Polym J 2017, 88, 575-585.

46. Hammer, D. A.; Discher, D. E., Synthetic cells-self-assembling polymer membranes and bioadhesive colloids. Annu Rev Mater Res 2001, 31, 387-404.

47. Itel, F.; Najer, A.; Palivan, C. G.; Meier, W., Dynamics of Membrane Proteins within Synthetic Polymer Membranes with Large Hydrophobic Mismatch. Nano Lett 2015, 15 (6), 3871-3878.

48. Lomora, M.; Dinu, I. A.; Itel, F.; Rigo, S.; Spulber, M.; Palivan, C. G., Does Membrane Thickness Affect the Transport of Selective Ions Mediated by Ionophores in Synthetic Membranes? Macromol Rapid Comm 2015, 36 (21), 1929-1934.

49. Rigler, P.; Meier, W., Encapsulation of fluorescent molecules by functionalized polymeric nanocontainers: Investigation by confocal fluorescence Imaging and fluorescence correlation Spectroscopy. J Am Chem Soc 2006, 128 (1), 367-373.

50. Hesse, M.; Heier, H.; Zeeh, B., Spektroskopische Methoden in der organischen Chemie. 6th ed; Thieme: 1979.

51. Craciun, I.; Denes, A. S.; Gunkel-Grabole, G.; Belluati, A.; Palivan, C. G., Surfaces Decorated with Polymeric Nanocompartments for pH Reporting. Helv Chim Acta 2018, 101 (3). 


\section{TOC Graphic}

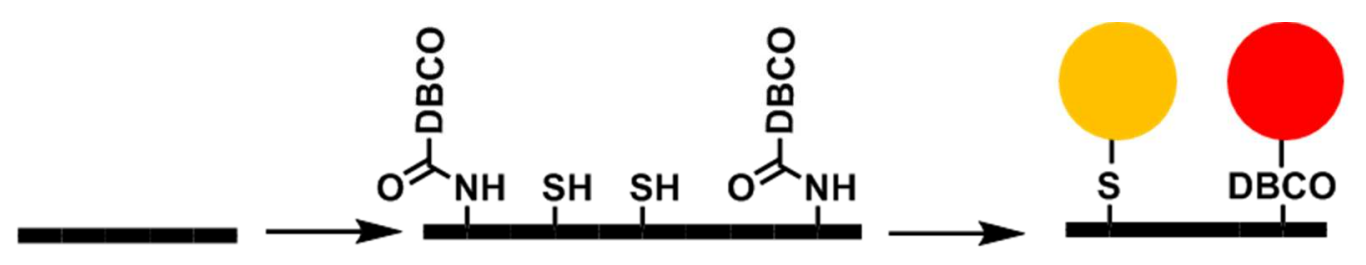

Silica Wafer
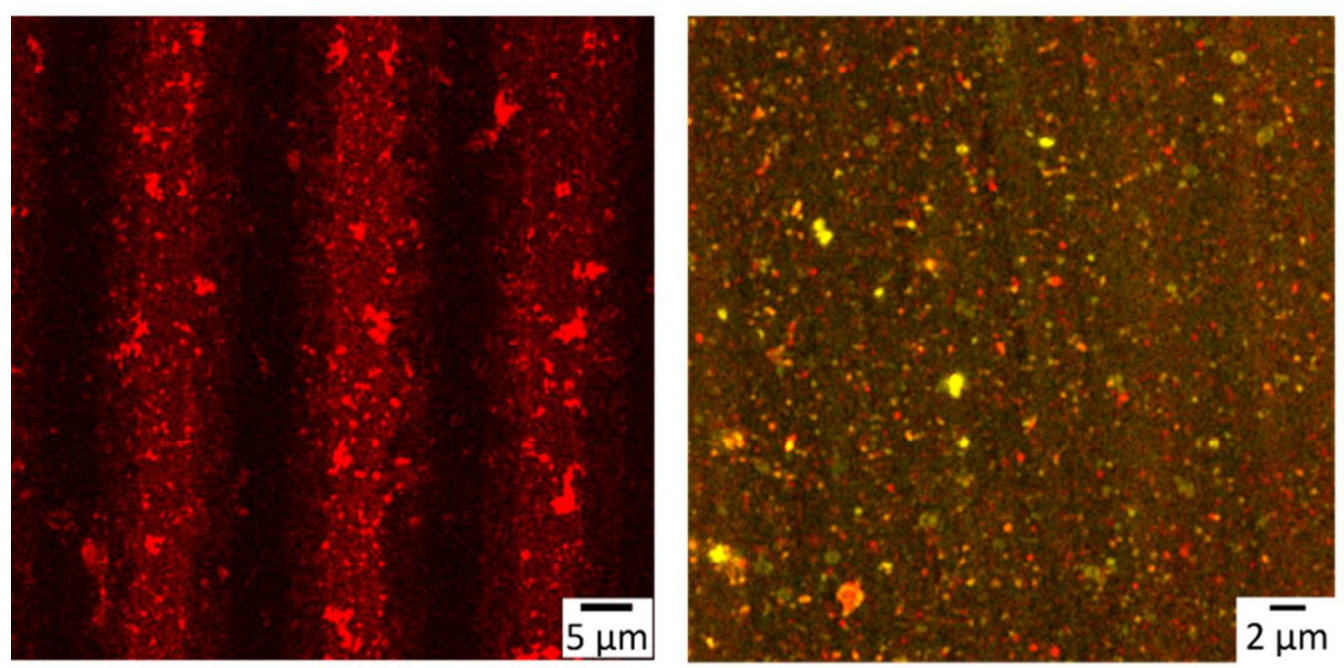

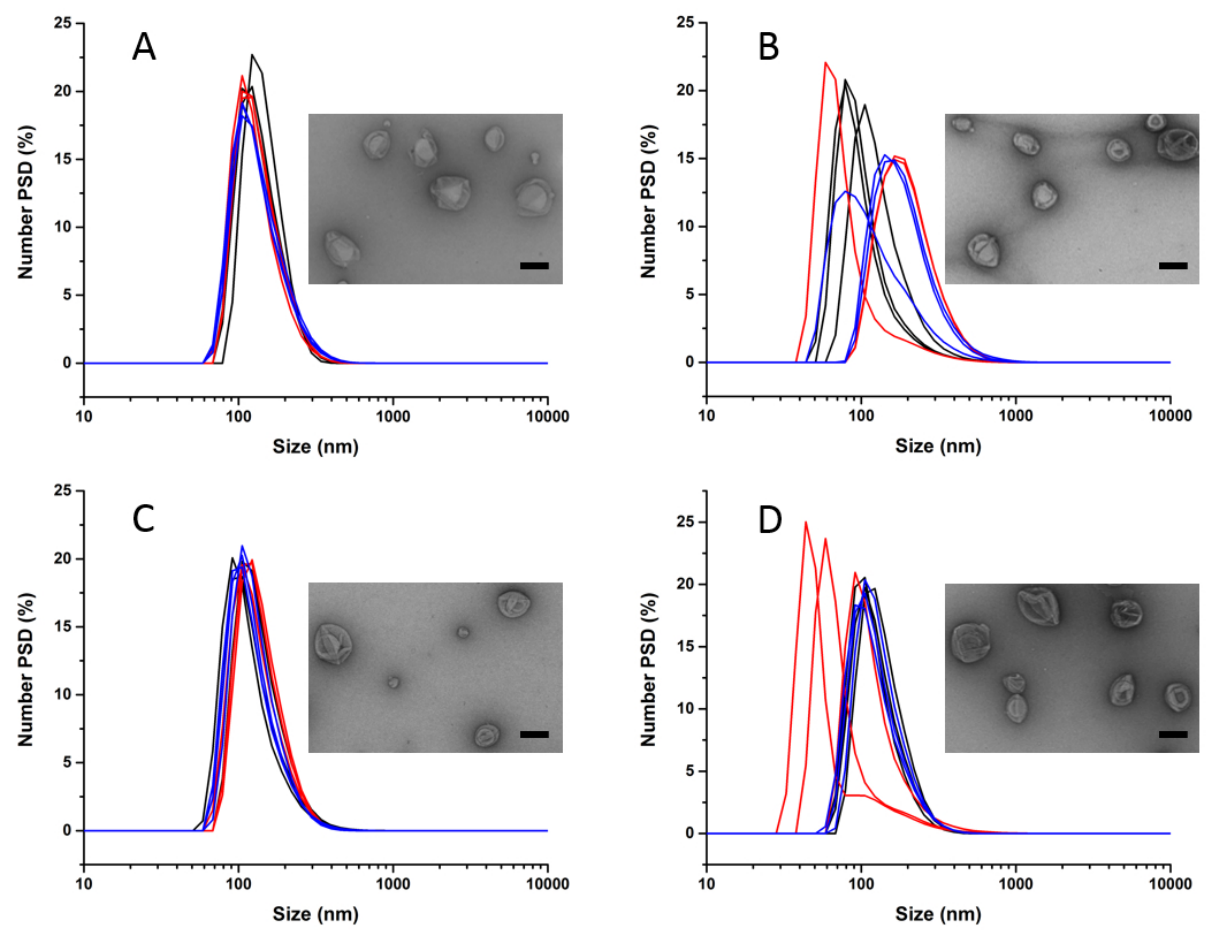

Figure 1. LS and TEM of azide-exposing nano-assemblies. DLS of polymersomes with different ratios of azide terminated polymers revealed similar diameters for the number average PSD: A) $0.0 \mathrm{~mol} \%, 139 \pm 4 \mathrm{~nm}$; B) $0.1 \mathrm{~mol} \%, 153 \pm 47 \mathrm{~nm}$; C) $1.0 \mathrm{~mol} \%, 132 \pm 7 \mathrm{~nm}$; D) $10 \mathrm{~mol} \%, 126 \pm 17 \mathrm{~nm}$. Three independent samples per polymersome ratio were measured three times each (black, red, and blue curves). Inserts are representative TEM images of the respective polymersomes, scale bars are $200 \mathrm{~nm}$.

$191 \times 145 \mathrm{~mm}(150 \times 150 \mathrm{DPI})$ 


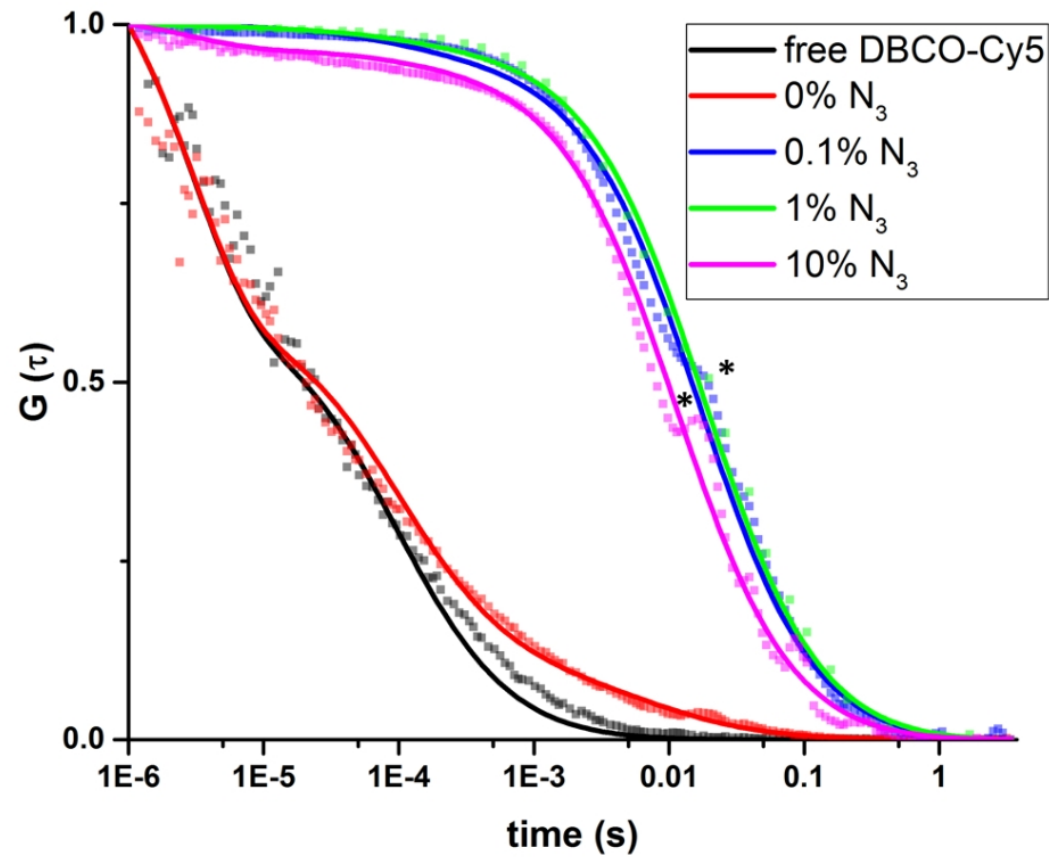

Figure 2. FCS of DBCO-Cy5 added to polymersomes containing different amounts of azide-terminated polymers. DBCO-Cy5 was added to the outside of the polymersomes and reacted overnight. The normalized autocorrelation curves for azide containing polymersomes showed a strong shift to higher diffusion times compared to azide free polymersomes (red line) or free DBCO-Cy5 (black line). The small step (*) in the autocorrelation curve is indicative for the presence of some clusters of polymersomes.

$205 \times 156 \mathrm{~mm}(150 \times 150 \mathrm{DPI})$ 
Figure 3. Schematic illustration of the two-step surface functionalization. B) Changes on static water contact angles of Si-Wafers during the different functionalization steps $(n=3)$. C-E) CLSM micrographs after reacting the surface during the different functionalization steps with Alexafluor488-azide: C) non-functionalized surface, D) NH2 functionalized surface, E) DBCO functionalized surface. Micrographs were recorded with the same settings and processed equally. Scale bars: $10 \mu \mathrm{m}$.

$175 \times 72 \mathrm{~mm}(150 \times 150 \mathrm{DPI})$ 
Figure 4. CLSM and AFM of immobilized polymersomes with different ratios of azide terminated polymers $\mathrm{A}$ ) $0.1 \mathrm{~mol} \% \mathrm{~B}) 1.0 \mathrm{~mol} \% \mathrm{C}) 10 \mathrm{~mol} \% \mathrm{D}) 0 \mathrm{~mol} \%$ on DBCO functionalized surfaces. Scale bars: $1 \mu \mathrm{m}$ for CLSM images. AFM scan size was $2.5 \mu \mathrm{m} \times 2.5 \mu \mathrm{m}$ each and the height scale bars are $70(A), 120$ (B), 70 (C) and $20 \mathrm{~nm}(\mathrm{D})$, respectively.

$178 \times 80 \mathrm{~mm}(150 \times 150 \mathrm{DPI})$ 


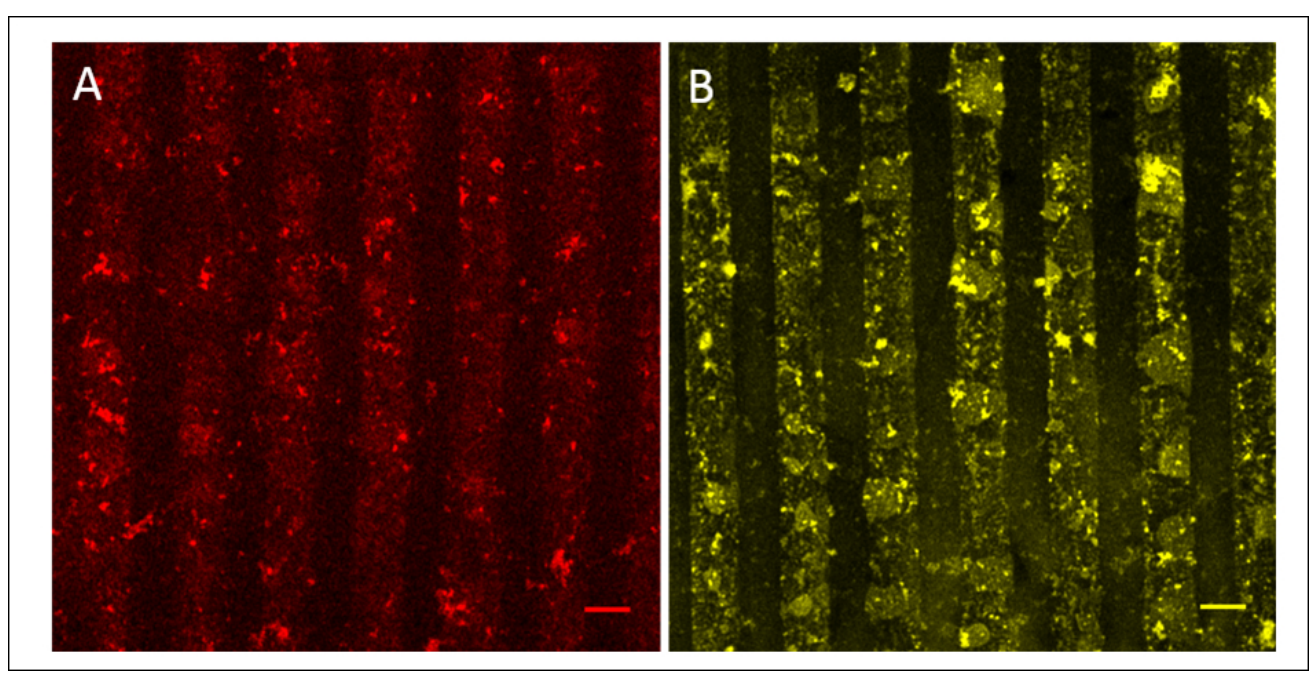

Figure 5. Polymersomes are immobilized within a pattern by $\mu \mathrm{CP}$ and observed with CLSM: A) Azidefunctionalized polymersomes stained with fluoresencent dye (Bodipy) and B) SRB labeled methacrylate functionalized polymersomes. Scale bars: $5 \mu \mathrm{m}$.

$160 \times 80 \mathrm{~mm}(150 \times 150 \mathrm{DPI})$ 


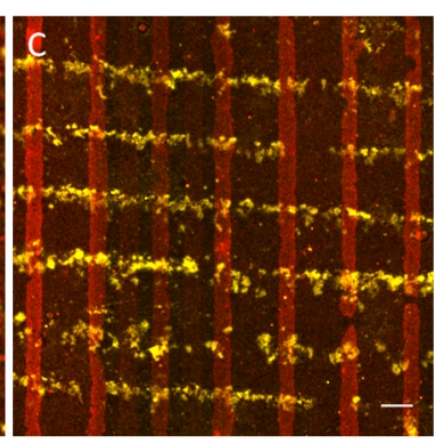

Figure 6. CLSM micrographs of azide functionalized polymersomes containing Bodipy co-immobilized with SRB labeled methacrylate functionalized nanostructures on DBCO and thiol bifunctionalized surfaces. A) Coimmobilization of azide-exposing polymersomes and methacrylate exposing polymersomes, B) Azideexposing polymersomes and methacrylate-exposing micelles and C) Checkerboard pattern obtained by first printing azide exposing polymerosomes and then methacrylate exposing micelles with the stamp rotated by $90^{\circ}$. Scale bars: A and B) $2 \mu \mathrm{m} \mathrm{C)} 5 \mu \mathrm{m}$. * hollow structure in the inset represents polymersomes and filled structures micelles.

$232 \times 82 \mathrm{~mm}(150 \times 150 \mathrm{DPI})$ 


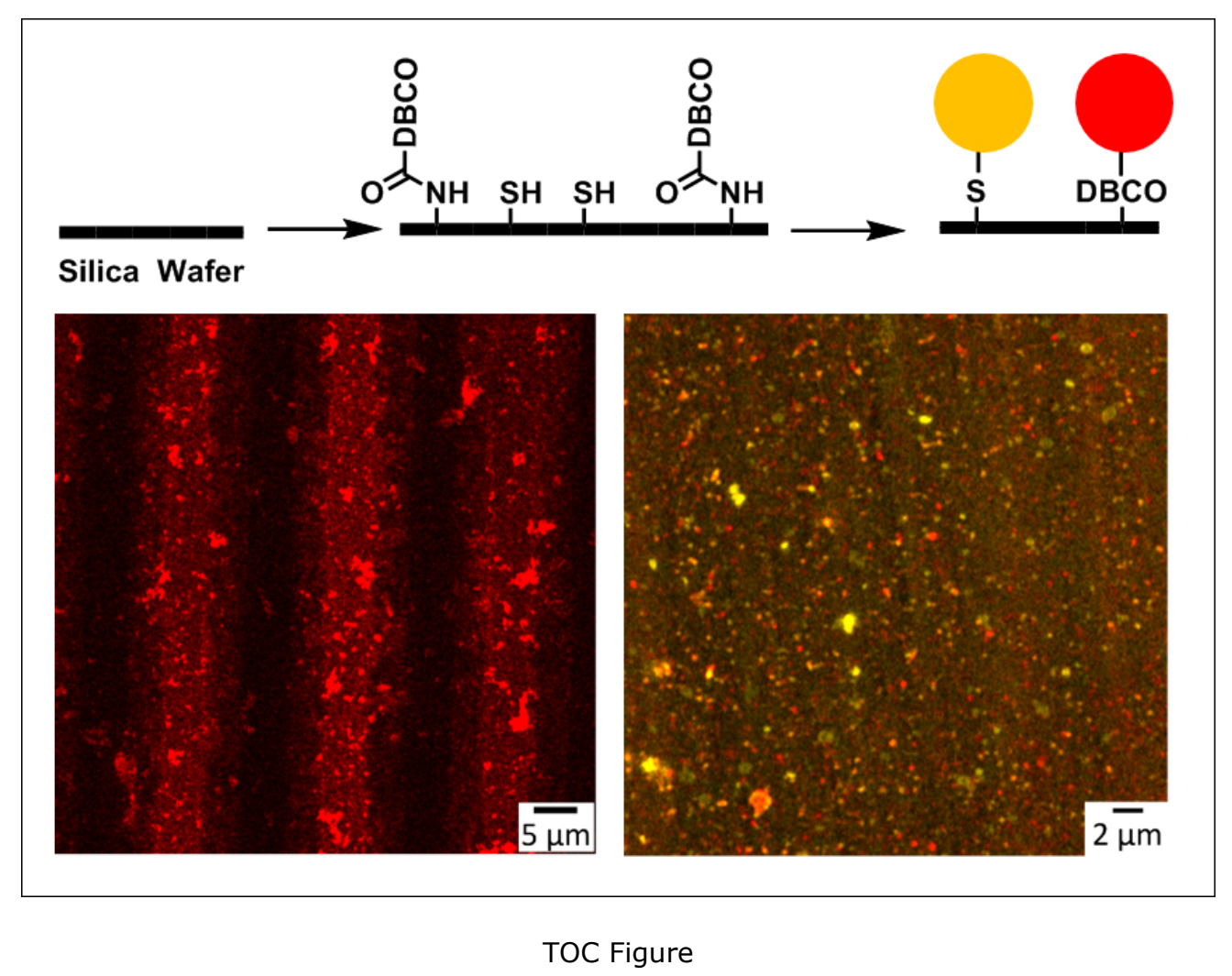

$144 \times 106 \mathrm{~mm}(150 \times 150 \mathrm{DPI})$ 